\title{
Simulation Modelling Application for Balancing Epidemic and Economic Crisis in the Region
}

\author{
Aleksey I. Borovkov ${ }^{1}$, Marina V. Bolsunovskaya ${ }^{1}$, Aleksei M. Gintciak ${ }^{1 *}$, Tatiana Ju. \\ Kudryavtseva ${ }^{1}$ \\ ${ }^{1}$ Peter the Great St. Petersburg Polytechnic University, 29 Polytechnicheskaya st., St. Petersburg 195251, \\ Russia
}

\begin{abstract}
The new coronavirus pandemic has had a major impact on worldwide economic development. Many infection-countering measures have imposed restrictions on economic activities. At the same time, economic sectors contribute unequally to both the disease's spread and to regional economic development. This article proposes a method for assessing the consequences of partial regional isolation. Simulation modelling and system dynamics are applied to assess both epidemiological and economic consequences. The classic "Susceptible - Exposed - Infected Recovered" disease spread model has been modified with the addition of a new group: Isolated Individuals. This modified model allows the size of the receptive population and the frequency of their contact to be regulated based on scenarios of partial regional isolation in the context of economic sectors. The model is tested in St. Petersburg. Changes in the region's infected population are forecasted as the result of the establishment and removal of partial isolation measures in the context of individual economic sectors.
\end{abstract}

Keywords: Economic crisis; Epidemic impact; Pandemic; Simulation modelling; System dynamics

\section{Introduction}

The new coronavirus (COVID-19) is an acute respiratory infection caused by the SARSCoV-2 virus (Rothan and Byrareddy, 2020). On March 11, 2020, a worldwide pandemic of the disease was declared. This state continues today, greatly impacting all spheres of human activity (Berawi, 2020). Many researchers are now predicting global changes to modern society as consequences of the pandemic (Tisdell, 2020). These estimates are based on two main approaches: extrapolation of the current dynamics of the pandemic's impact, and translation of the effects of previous epidemics and pandemics to the current situation.

Extrapolating the current pandemic's impact on changes to modern society may not be appropriate because of the complexity of society as a system. Public relations in different spheres are characterized by their nonlinearity, the presence of feedback loops, inaccurate parameters, and openness and delays in system response. These factors mean that any extrapolations concerning current impact can only be applied in the very short term. It is impossible to estimate the absolute or relative magnitude of influence using this approach, as the pandemic is not yet over.

*Corresponding author's email: gintsyak_am@spbstu.ru, Tel.: +7-911-157-74-26 doi: 10.14716/ijtech.v11i8.4529 
In the 21st century alone, society has experienced several global disease outbreaks: the SARS-CoV pandemic in 2002-2003, the H1N1 influenza pandemic in 2009-2010, and the Ebola epidemic in 2014-2016. These cases, however, are inferior to the current COVID-19 pandemic either in their globality (as in the case of Ebola) or lethality (as in the cases of H1N1 influenza and SARS-CoV). In addition, society has changed since these events: behavioral habits adopted by some of the population, for example, affect both the spread of the virus and acceptance of the pandemic's impact. Still, some studies have potential usefulness in assessing the impact of the current pandemic (Smith et al., 2009).

Several studies report that the global economy has historically been most influenced by epidemics and pandemics. This is confirmed by current research (Baldwin and Weder, 2020). The named key factors of a pandemic's impact on the economy are border closures, damage to global logistics, reduced consumption of specific goods, and the downtime of production capacity. Further, these consequences affect different economy sectors unevenly.

Some scientists (Fernandes, 2020) conclude that the damage to the economy and society from measures designed to counter the spread of a virus may exceed the damage caused by the disease itself. Consequently, there is a need to balance epidemiological and economic damage while strategies are being formed to counter regional spread.

On the one hand, government measures should sufficiently contain the incidence of a disease like COVID-19 at such a level that the healthcare system is able to cope with active patients. This capability requires sufficient medical personnel with the needed qualifications, certain quantities of specialized equipment, and the ready availability of personal protective equipment and consumable medical supplies. Stricter containment measures may also reduce the total number of cases and the number of active patients, thereby reducing the burden on the healthcare system. On the other hand, the measures countering the spread of the disease must be structured to reduce the pandemic's negative economic (both global and regional) and societal impacts (Shirov, 2020).

Strict containment measures reduce the consumption of certain goods and services, increase the cost of production, and contribute to the forced downtime of enterprises. This makes the development and implementation of measures to counter the spread of COVID19 an optimization task. Existing empirical approaches do not account for the complexity of the region as a system; as such, any results of management decisions based on an empirical approach are far from optimal values. This demonstrates the need for a scientific approach to assessing the epidemiological and economic implications of the various strategies designed to counter the regional spread of an infectious disease. To do so, this study proposes a model that allows regional morbidity dynamics to be predicted in relation to the results of partial isolation in the context of economic sectors.

\section{Methods}

\subsection{Simulation Modelling}

Simulation modelling is proposed as the preferred method for investigating the impact of strategic economic isolation in a given region in the incidence of viral infection. Simulation modelling is a method of research in which the system studied is replaced by a model describing the real system with a sufficient degree of accuracy (Rodič, 2017; Tsvetkova et al., 2017). Using the developed model, simulation experiments are conducted to assess the behavior of the real system in similar situations.

The ability to predict the behavior of a real system through simulations depends on the accuracy and adequacy of the assumptions used in the development of the model. Accounting for all the factors affecting the system is fundamentally impossible (Rodič, 
2017), so the researcher needs to transfer to the model those parameters that are essential within the framework of the current study.

Simulation modelling is the most appropriate way to study the spread of infectious diseases in the absence of complete and accurate information about the disease's properties (Currie et al., 2020). Using this method of research allows the nature of a virus to be studied and the dynamics of its spread under different scenarios of event development to be predicted (Giordano et al., 2020). The daily number of new and active infectious individuals are the main data used by the researchers in the present study for the specified purposes.

\subsection{System Dynamics}

System dynamics are used when creating highly abstract simulations of social engineering systems (Husin et al., 2015; Upachaban et al., 2016). As such, they are what tends to be used as a simulation paradigm to model trends like the spread of infectious diseases (Mecoli et al., 2013; Upadhyay and Roy, 2014). The approach is based on a continuous change in system indicators over time. In this case, the simulation model is set by a system of differential equations linking the integral and derived indicators of the system. The simulation experiment consists of the numerical integration of a system of differential equations for various initial indicator values.

System dynamics is applicable in the present study for several reasons. First, the paradigm supports the possibility of using complex cause-and-effect relationships and dependencies, including positive and negative feedback loops. The presence of such loops is characteristic of society as a complex system. Second, the incubation period of infectious disease can be modelled via system dynamics in the form of a delay in the response of the system. Third, system dynamics enable using an interval approach to model the system in the absence of complete and accurate information (i.e., the properties of the disease) to determine system indicators.

\subsection{SIR-model and Its Extensions}

The Susceptible - Infected - Recovered (SIR) class of models is widely known (Ma et al., 2004). Under the basic SIR model, the population of a region or other simulated object is divided into three groups: susceptible (S), infected (I), and recovered (R). The number of individuals belonging to a particular group changes over time according to the specified characteristics of dependencies. Typically, the rate of transition of individuals from the susceptible group to the infected group is affected by the frequency of contact between individuals, the virality of the disease, and the number of susceptible and infected individuals at the present time (Osthus et al., 2017). The speed of transition from the infected to the recovered state is affected by the illness' average duration.

There are many modifications of the SIR model. For example, Susceptible - Infected Susceptible (SIS) class models are used to simulate the spread of diseases in cases where affected individuals are not immune to reinfection (Qi et al., 2017). There are also Susceptible - Infected - Recovered - Susceptible (SIRS) models, in which recovered individuals obtain immunity but gradually lose it due to virus mutations or other reasons (Li et al., 2017). Susceptible - Infected - Recovered - Dead (SIRD) models are designed to simulate the mortality of individuals as a result of disease (Lalwani et al., 2020).

To predict the dynamics of COVID-19, this study recommends another modification of the SIR-model: Susceptible - Exposed - Infected - Recovered (SEIR) (Li et al., 1999). This accounts for the addition of a group of exposed (i.e., in the incubation period) individuals. As the long incubation period is a key feature of COVID-19 (Lauer et al., 2020), the use of this model is justified. To use system theory terminology, a long incubation period is a delay in the system's response. This means that any impacts aimed at changing the current 
incidence of the disease can affect the total number of infected and the current number of active patients only after a certain period.

The classic SEIR model is described by a system of differential equations:

$$
\begin{gathered}
\frac{d S(t)}{d t}=-\beta S I \\
\frac{d E(t)}{d t}=\beta S I-\gamma E \\
\frac{d I(t)}{d t}=\gamma E-\delta I \\
\frac{d R(t)}{d t}=\delta I
\end{gathered}
$$

where $S(t)$ is the number of susceptible individuals at a time $t ; E(t)$ is the number of exposed individuals at a time $t ; I(t)$ is the number of infected individuals at a time $t ; R(t)$ is the number of recovered individuals at a time $t ; \beta$ is the individuals' contact rate with subsequent infection; $\gamma$ is the rate of the transition of individuals to the stage of infection (the value inverse to the average duration of the incubation period); and $\delta$ is the recovery rate for infected individuals (the value inverse to the mean duration of the disease).

Figure 1 presents a structural scheme of the classic SEIR model.

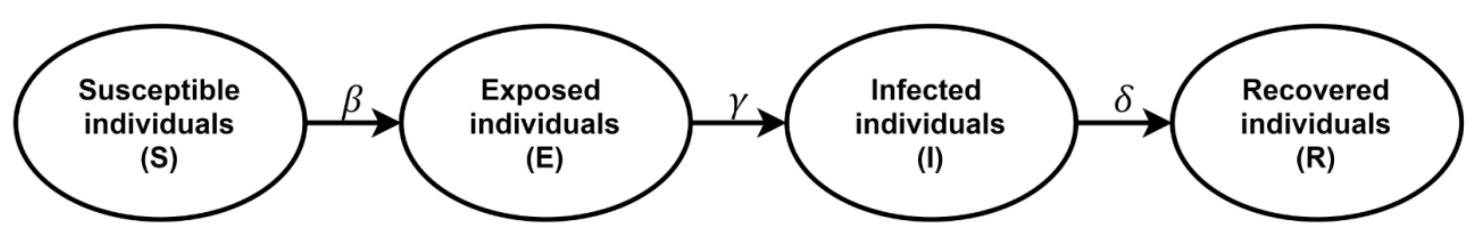

Figure 1 Structure of the SEIR model

The classic SEIR model allows disease spread to be simulated in absolute conditions in the absence of external influence from executive authorities. It does not factor in the possible isolation of individuals, the introduction of quarantines, the use of personal protective equipment, or social distancing. As a result, this model can be successfully applied to study the nature of the virus and predict its short-term consequences in the absence of any measures to counter its spread. However, this also means that the model is inapplicable for drafting proposals and testing hypotheses for the adoption of control measures.

\subsection{Improvement of the Traditional SEIR Model}

For the present study, the classic SEIR model is expanded to include another group: individuals in isolation or quarantine. These individuals are segregated from the receptive individuals. The modelling assumes that individuals from this added group do not participate in social interaction and do not have the opportunity to be infected.

Before starting the modelling, the rules by which individuals move between the receptive and isolated groups must be determined. Several approaches may be used to accomplish this. The intensity of the transition between the groups in both directions can be viewed as continuous functions, depending on the current number of infected people and the psychoemotional state of the population against the background of a pandemic. Another approach involves a discrete form of the transition rate function corresponding to the directive scenarios for the introduction and removal of control measures. Such 
scenarios can include isolation or the lifting of regional isolation measures as related to individual economic sectors.

The developed modification of the SEIR model is described by the following system of differential equations:

$$
\begin{gathered}
\frac{d S(t)}{d t}=-\beta S I-\varphi(t)+\omega(t) \\
\frac{d Q(t)}{d t}=\varphi(t)-\omega(t) \\
\frac{d E(t)}{d t}=\beta S I-\gamma E \\
\frac{d I(t)}{d t}=\gamma E-\delta I \\
\frac{d R(t)}{d t}=\delta I
\end{gathered}
$$

where $Q(t)$ is the number of healthy individuals in quarantine and isolation at the time $t$; $\varphi(t)$ is a function corresponding to the scenario of the introduction of isolation (quarantine) measures for healthy people; and $\omega(t)$ is a function corresponding to the scenario of lifting isolation (quarantine) measures for healthy people.

Figure 2 presents a structural scheme of the modified SEIR model.

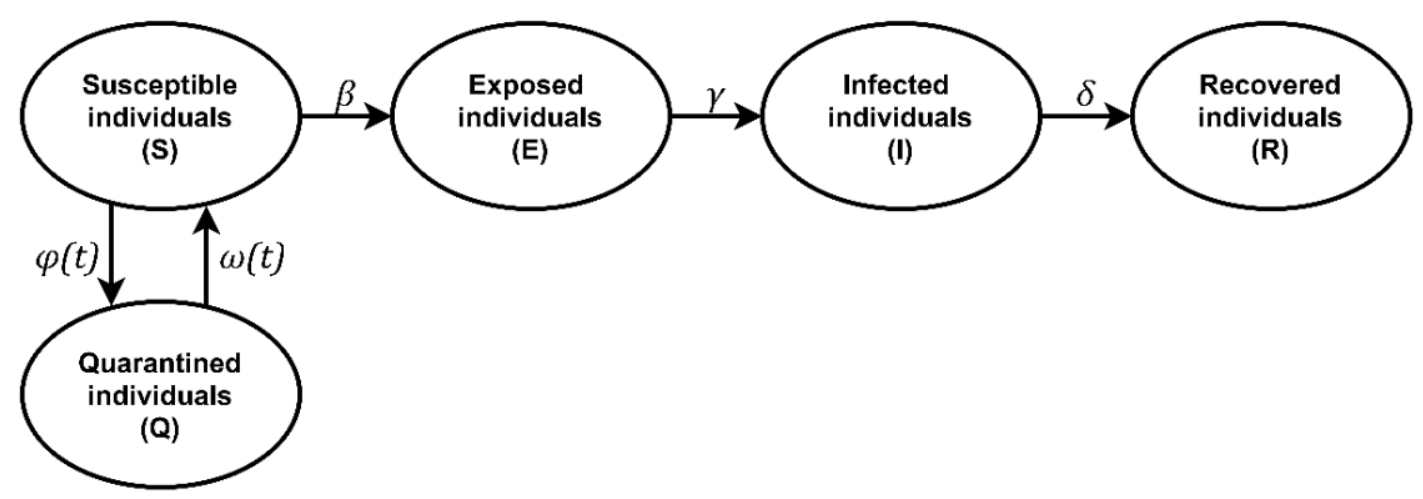

Figure 2 Structure of the modified SEIR model with quarantined individuals (SQEIR)

This modification of the SEIR model allows the number of susceptible individuals available for infection during modelling to be adjusted according to isolation scenarios (i.e., measures designed to prevent susceptible people from becoming infected). This means the model becomes more accurate to the real system, as the reduction of individuals in contact during the pandemic is objectively present in society. Moreover, the modification creates the possibility for scenario modelling. Scenario modelling involves assessing the epidemiological consequences of various strategies for countering the spread of an infectious disease in a given region.

The study assumes that scenarios for the introduction and lifting of localized isolation concerning specific economic sectors are consistently determined by regional executive authorities, while the functions corresponding to the scenarios of introducing and lifting isolation (quarantine) measures have the following forms:

$$
\varphi(t)=\left\{\begin{array}{c}
p_{i}, \text { if } t=\tau_{i} \\
0, \text { if } t \neq \tau_{i}
\end{array}\right.
$$




$$
\begin{gathered}
\omega(t)=\left\{\begin{array}{c}
p_{i}, \text { if } t=\sigma_{i} \\
0, \text { if } t \neq \sigma_{i}
\end{array}\right. \\
i \in\{1, \ldots, n\}
\end{gathered}
$$

where $n$ is the number of sectors of the economy that are subject to local isolation (quarantine) in the region; $p_{i}$ is the number of people employed in the ith sector of the economy; $\tau_{i}$ is the moment in which the $i$ th sector of the economy introduces its mode of local isolation (quarantine); and $\sigma_{i}$ is the moment in time at which the local isolation (quarantine) regime is lifted in the ith sector of the economy. The model is indifferent to the isolation of infected people as the Quarantined group does not suppose the interaction of people inside.

\subsection{Initial Data}

St. Petersburg has been chosen as the setting for demonstrating the model. According to the Federal State Statistics Service, the population of St. Petersburg is 5.38 million, with 3.06 million part of the workforce.

Table 1 lists the employment structures in St. Petersburg by economic sector in 2019. Labor distribution data were obtained from official state statistics sources. All sectors of the economy where the number of employed exceeds 20,000 have been accounted for.

Table 1 Distribution of employment in St. Petersburg by industry

\begin{tabular}{clc}
\hline No. & \multicolumn{1}{c}{ Industry } & Employed, $10^{3}$ \\
\hline 1 & Retail and wholesale & 664.9 \\
2 & Manufacturing & 462.7 \\
3 & Construction & 319.1 \\
4 & Logistics & 273.2 \\
5 & Education & 236.3 \\
6 & Healthcare & 167.2 \\
7 & Real estate & 115.4 \\
8 & Services & 108.1 \\
- & Other & 712.9 \\
\hline
\end{tabular}

This distribution of employment in St. Petersburg by industry is used to form scenarios for introducing and lifting measures designed to counter the spread of COVID-19.

\section{Results and Discussion}

The simulation experiments use the characteristics of the infectious disease identified by the scientific community in the initial stages of its spread (Lauer et al., 2020). The simulation is performed for different types of functions that correspond to the introduction and lifting of isolation (quarantine) measures. Table 2 describes the simulation experiments conducted using the modified SEIR model. A set of six experiments was sufficient to assess the impact of quarantine measures. The initial data for each experiment include isolated industries that indicate the timing of the introduction and lifting of isolation measures (industry numbers correspond to the rows in Table 1). The output data are as follows:

- the maximum number of infected patients during the pandemic (consistent with the burden on the healthcare system);

- the total number of recovered individuals (can be used to estimate mortality from the infectious disease); 
- the day when the number of people infected will fall below the threshold of $0.01 \%$ of the total population (consistent with the possibility of an early transition to normal life);

- the total number of man-days spent in isolation (consistent with the negative economic effect of local isolation of economic sectors).

Table 2 Simulation experiments results

\begin{tabular}{cccccccc}
\hline Exp. & $\begin{array}{c}\text { Isolated } \\
\text { industries }\end{array}$ & Start & End & $\begin{array}{c}\text { Max. } \\
\text { infected }\end{array}$ & $\begin{array}{c}\text { Total } \\
\text { infected }\end{array}$ & $\begin{array}{c}\text { Day No. } \\
\text { infected }<0.01 \%)\end{array}$ & $\begin{array}{c}\text { Workdays in } \\
\text { isolation }\end{array}$ \\
\hline 1 & - & - & - & $8.46 \times 10^{5}$ & $3.40 \times 10^{6}$ & 202 & 0 \\
\hline 2 & $1,5,8$ & 14 & 112 & $1.36 \times 10^{5}$ & $7.03 \times 10^{5}$ & 204 & $1.29 \times 10^{8}$ \\
\hline 3 & $\begin{array}{c}1,2,4, \\
5,7,8\end{array}$ & 14 & 112 & $1.13 \times 10^{4}$ & $6.12 \times 10^{4}$ & 166 & $2.37 \times 10^{8}$ \\
\hline 4 & $\begin{array}{c}1,2,3,4, \\
5,7,8\end{array}$ & 14 & 112 & $4.03 \times 10^{3}$ & $2.17 \times 10^{4}$ & 142 & $2.78 \times 10^{8}$ \\
\hline 5 & $\begin{array}{r}1,2,4, \\
5,7,8\end{array}$ & 0 & 98 & $1.48 \times 10^{3}$ & $8.93 \times 10^{3}$ & 136 & $2.37 \times 10^{8}$ \\
\hline 6 & $1,2,4$, & 28 & 126 & $5.19 \times 10^{4}$ & $2.66 \times 10^{5}$ & 182 & $2.37 \times 10^{8}$ \\
\hline
\end{tabular}

The inputs of experiment No. 3 (Table 2) correspond with the measures taken by the Government of St. Petersburg. The dynamics of the spread of the disease in experiment No. 3 correspond to those of the spread of COVID-19 in St. Petersburg from March to June 2020, according to official data ( $\mathrm{SD}=1.52 \%$ ). The isolated industries for experiments No. 2 and No. 4 are taken in more or less quantity to allow infection spread dynamics to be compared. Experiments No. 5 and No. 6 differ from No. 3 in terms of when the countering measures were introduced. Input data (isolated industries, start and end of isolation) are used in the model through Equations 10 and 11.

The first group of experiments (No. 1-4) was performed to assess the impact of a set of isolated industries on the dynamics of the spread of COVID-19. Experiment No. 1 did not involve isolating any branches of the economy, so it served as the basis for comparison. The results of this first group demonstrate that the dynamics of the virus' spread have a nonlinear dependence on the number of workers employed in the isolated sectors of the economy. For example, the isolation of three economic sectors, which make up approximately $42 \%$ of the total number of workers (experiment No. 2), reduces the peak number of infected people by 6.2 times and the total number of patients by 4.83 times. Isolation of six sectors of the economy, accounting for $80 \%$ of the total number of workers (experiment No. 3), reduces the peak number of infected people by 75 times and the total number of recovered individuals by 56 times.

The second group of experiments (No. 3, No. 5-6) was conducted to assess the impact of the rate of isolation of economic sectors on the dynamics of the virus' spread. Experiment No. 3 corresponds to actual measures taken in St. Petersburg. Experiments No. 5 and No. 6 present alternative scenarios of events, including the isolation of similar sectors of the economy, but 14 days earlier and later, respectively. Figure 3 presents the results of the second group of experiments. The experiments differ in terms of the day that isolation was introduced, so the graphs comparison can be used to define the Quarantine impact. The xaxis represents the days of infection spread, and the y-axis the numbers of infected people.

The results of the second group show that the introduction of measures to isolate the economy and public life in the early stages of virus' spread can reduce the incidence of the 
disease and bring an earlier end to the pandemic. Conversely, the late introduction of isolation measures affects the dynamics of the disease's spread to a lesser extent. These results support assessments made by leading epidemiologists (Giordano et al., 2020).

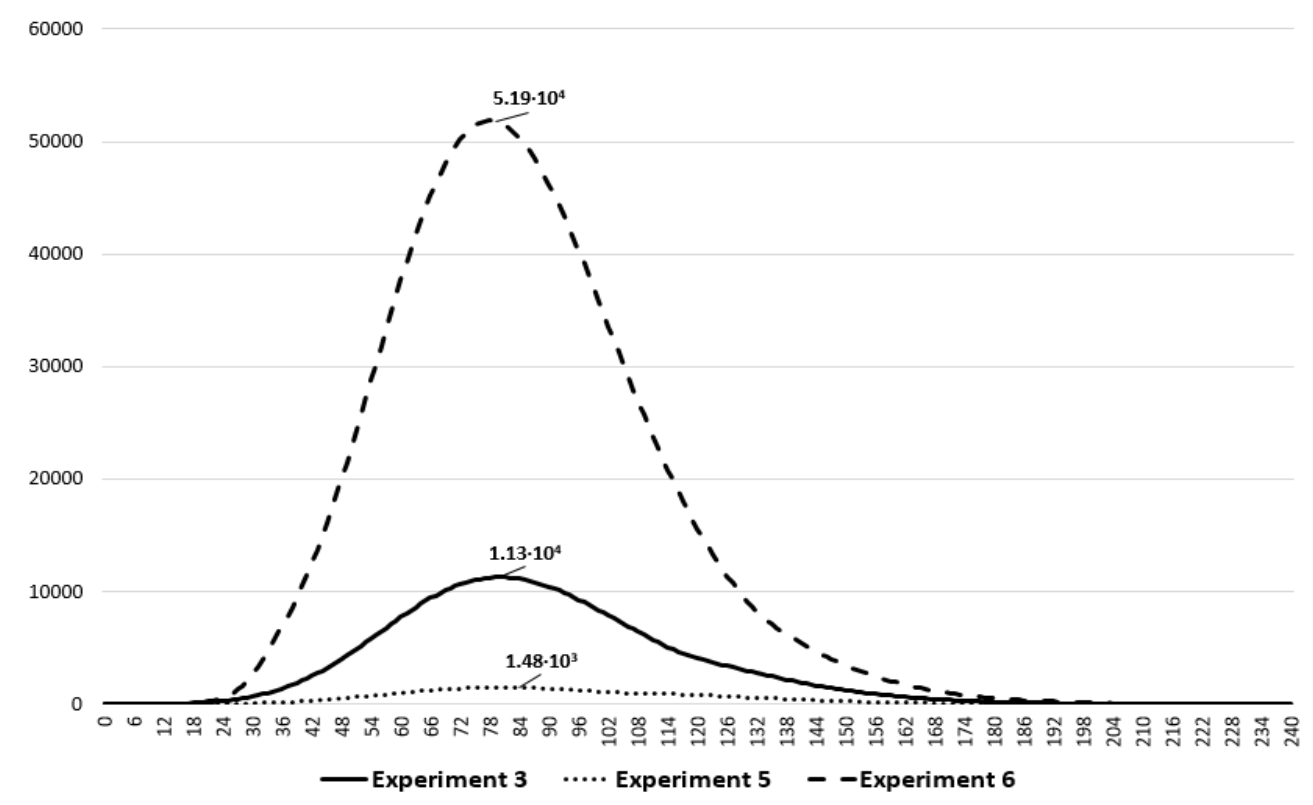

Figure 3 Results of experiments No. 3, 5 and 6

In the case of an unstable system, early measures can most affect system dynamics. The present study's experimental results confirm this statement. Epidemiological impact grows multiple times if the control measures are postponed for weeks. Conversely, economic impact linearly depends only on the duration of isolation and the number of workers isolated. These results indicate that the optimal disease-countering strategy is total isolation as early as possible.

The present study's modified version of the SEIR model can be used to develop strategies for countering the spread of infectious diseases. It also enables researchers to assess the possible mortality in each case as a proportion of the total infected and recovered population. However, the assumptions used in the model's development must be factored in.

First, this model assumes that the impact of isolating certain sectors of the economy and public life depends only on the interaction of employees in the workplace. It does not consider professional activities that necessitate direct communication among people (e.g., the service sector, catering, education) (Redko et al., 2020). Isolating these economic sectors would likely influence the disease's spread to a greater degree (Fernandes, 2020).

Further, the proposed model considers only the complete isolation of economic sectors. In reality, partial isolation also takes place (Baldwin and Weder, 2020). This limitation of the model can be overcome by splitting large sectors of the economy into smaller ones.

Another significant limitation of the model is its disregard for the behavioral characteristics of the population. Under system dynamics models, individuals are unable to voluntarily comply with or violate the isolation measures imposed. Opportunities to account for such behavior could be presented by agent-based models (Silva et al., 2020). Such models focus on individual agents' behavioral characteristics, which are superfluous when observing disease spread dynamics on a regional scale. 


\section{Conclusions}

This article develops a methodological apparatus of an SIR-class model for practical use in decision-making by regional leaders. The proposed modified SQEIR model allows researchers to form proposals and test hypotheses concerning the different control measures adopted in specific regions.

According to the proposed approach, various strategies for countering the spread of an infectious disease in an economic context are transformed into a modelling scenario. The series of simulation experiments produce a predictive series that can describe the dynamics of the spread of an infectious disease. Based on these data series, it is possible to estimate the potential mortality in a given region, the burden on the healthcare system, and the economic damage likely to result.

The proposed approach may be used to estimate the spread of COVID-19 infection in 10 regions of the Russian Federation, including Moscow and St. Petersburg, from March 2020 to the present (December 2020). Governments may then use these modeling results to form infection-countering strategies. It is hoped that this model will help decisionmakers find a balance between the negative effects of the virus' spread and the economic damages resultant from countermeasures.

\section{Acknowledgements}

This research is partially funded by the Ministry of Science and Higher Education of the Russian Federation as part of the World-Class Research Center Program: Advanced Digital Technologies (contract No. 075-15-2020-934 dated 17.11.2020). The research is funded by the Russian Science Foundation (project No. 20-78-10123).

\section{References}

Baldwin, R.E., Weder, B., 2020. Economics in the Time of COVID-19. Centre for Economic Policy Research. Available Online at https://voxeu.org/system/files/epublication/COVID-19.pdf, Accessed on November 15,2020

Berawi, M.A., 2020. Empowering Healthcare, Economic, and Social Resilience during Global Pandemic Covid-19. International Journal of Technology, Volume 11(3), pp. 436-439

Currie, C.S.M., Fowler, J.W., Kotiadis, K., Monks, T., Onggo, B.S., Robertson, D.A., Tako, A.A., 2020. How Simulation Modelling Can Help Reduce the Impact of COVID-19. Journal of Simulation, Volume 14(2), pp. 83-97

Fernandes, N., 2020. Economic Effects of Coronavirus Outbreak (COVID-19) on the World Economy. IESE Business School Working Paper No. WP-1240-E, Available Online at https://ssrn.com/abstract=3557504 or http://dx.doi.org/10.2139/ssrn.3557504

Giordano, G., Blanchini, F., Bruno, R., Colaneri, P., Di Filippo, A., Di Matteo, A., Colaneri, M., 2020. Modelling the COVID-19 Epidemic and Implementation of Population-wide Interventions in Italy. Nature Medicine, Volume 26, pp. 855-860

Husin, A.E., Berawi, M.A., Dikun, S., Ilyas, T., Berawi, A.R.B., 2015. Forecasting Demand on Mega Infrastructure Projects: Increasing Financial Feasibility. International Journal of Technology, Volume 6(1), pp. 73-83

Lalwani, S., Sahni, G., Mewara, B., Kumar, R., 2020. Predicting Optimal Lockdown Period with Parametric Approach using Three-phase Maturation SIRD Model for COVID-19 Pandemic. Chaos, Solitons \& Fractals, Volume 138, pp. 1-8

Lauer, S.A., Grantz, K.H., Bi, Q., Jones, F.K., Zheng, Q., Meredith, H.R., Azman, A.S., Reich, N.G., Lessler, J., 2020. The Incubation Period of Coronavirus Disease 2019 (COVID-19) From 
Publicly Reported Confirmed Cases: Estimation and Application. Annals of Internal Medicine, Volume 172(9), pp. 577-582

Li, D., Liu, S., Cui, J., 2017. Threshold Dynamics and Ergodicity of an SIRS Epidemic Model with Markovian Switching. Journal of Differential Equations, Volume 263(12), pp. 8873-8915

Li, M.Y., Graef, J.R., Wang, L., Karsai, J., 1999. Global Dynamics of a SEIR Model with Varying Total Population Size. Mathematical Biosciences, Volume 160(2), pp. 191-213

Ma, W., Song, M., Takeuchi, Y., 2004. Global Stability of an SIR Epidemic Model with Time Delay. Applied Mathematics Letters, Volume 17(10), pp. 1141-1145

Mecoli, M., De Angelis, V., Brailsford, S.C., 2013. Using System Dynamics to Evaluate Control Strategies for Mosquito-Borne Diseases Spread by Human Travel. Computers \& Operations Research, Volume 40(9), pp. 2219-2228

Osthus, D., Hickmann, K.S., Caragea, P.C., Higdon, D., Del Valle, S.Y., 2017. Forecasting Seasonal Influenza with a State-Space SIR Model. The Annals of Applied Statistics, Volume 11(1), pp. 202-224

Qi, H., Liu, L., Meng, X., 2017. Dynamics of a Nonautonomous Stochastic SIS Epidemic Model with Double Epidemic Hypothesis. Complexity, Volume 2017, pp. 1-14

Redko, S.G., Tsvetkova, N.A., Seledtsova, I.A., Golubev, S.A., 2020. Systematic Approach to Education of Specialists for a New Technological Paradigm. In: Arseniev, D.G., Overmeyer, L., Kälviäinen, H., Katalinić, B., (Eds.), Cyber-Physical Systems and Control, Springer International Publishing, Cham, pp. 643-650

Rodič, B., 2017. Industry 4.0 and the New Simulation Modelling Paradigm. Organizacija, Volume 50(3), pp. 193-207

Rothan, H.A., Byrareddy, S.N., 2020. The Epidemiology and Pathogenesis of Coronavirus Disease (COVID-19) Outbreak. Journal of Autoimmunity, Volume 109, pp. 1-4

Shirov, A.A., 2020. Statistics for the Benefit of Economics and Society. Studies on Russian Economic Development, Volume 31, pp. 3-6

Silva, P.C.L., Batista, P.V.C., Lima, H.S., Alves, M.A., Guimarães, F.G., Silva, R.C.P., 2020. COVIDABS: An Agent-Based Model of COVID-19 Epidemic to Simulate Health and Economic Effects of Social Distancing Interventions. Chaos, Solitons \& Fractals, Volume 139, pp. 1-15

Smith, R.D., Keogh-Brown, M.R., Barnett, T., Tait, J., 2009. The Economy-Wide Impact of Pandemic Influenza on the UK: A Computable General Equilibrium Modelling Experiment. BMJ, Volume 339, pp. b4571-b4571

Tisdell, C.A., 2020. Economic, Social and Political Issues Raised by the COVID-19 Pandemic. Economic Analysis and Policy, Volume 68, pp. 17-28

Tsvetkova, N.A., Tukkel, I.L., Ablyazov, V.I., 2017. Simulation Modeling the Spread of Innovations. In: 2017 XX IEEE International Conference on Soft Computing and Measurements (SCM), St. Petersburg, pp. 675-677

Upachaban, T., Khongsatit, K., Radpukdee, T., 2016. Mathematical Model and Simulation Study of a Closed-Poultry House Environment. International Journal of Technology, Volume 7(7), pp. 1246-1252

Upadhyay, R.K., Roy, P., 2014. Spread of a Disease and its Effect on Population Dynamics in an Eco-Epidemiological System. Communications in Nonlinear Science and Numerical Simulation, Volume 19(12), pp. 4170-4184 